\title{
The National Bureau of Standards Gas Thermometer II. Measurement of Capacitance to a Grounded Sur- face With a Transformer Ratio-Arm Bridge
}

\author{
L. A. Guildner and R. E. Edsinger
}

(September 4, 1964)

\begin{abstract}
A modification of the conventional transformer ratio-arm capacitance bridge was made to measure the values of three-lead capacitors having one of the plates grounded. With this ground point it is necessary to use triaxial cable with solid shields. A troublesome capacitance coupling between the primary and secondary shields of the transformer was neutralized by means which still enable one to attain the extremely high precision and accuracy characteristic of this type of bridge. The modified bridge was developed initially for highly accurate determinations of the locations of the grounded mercury menisci of the National $\mathrm{Bu}-$ reau of Standards precision manometer. Other applications are readily thought of and one interesting example is presented.
\end{abstract}

\section{Introduction}

In the last decade, one of the important developments in the field of metrology is the recognition of the value of transformer ratio-arm bridges using 3lead capacitors. $[1,2,3,4] .{ }^{1}$ 'This system can measure capacitance without including parasitic lead eftects, and is capable of such accuracy for a computable $1 \mathrm{pF}$ capacitor, $[6,7]$, that it is used to check the U.S. standards of the electrical units. Not only is the bridge system essentially simple, but it has a special elegance in the ability to produce voltages in ratios which are very precisely the turns ratios of the transformer secondary windings. Thus a decade system or any other ratio of integral numbers within the limits of a practical number of turns may readily be established.

When conditions permit, both plates of the capacitors are isolated and the bridge is grounded at the junction of the ratio-arm secondary windings. However, under a variety of conditions it is at least very inconvenient, if not impossible, to prevent one of the plates of the capacitor from being grounded. In particular, many opportunities arise for computing very small distances or changes of distances from capacitance measurements, where the object under observation is grounded.

This paper presents the modifications for changing the ground connection from the center-tap of the ratio-arms of the bridge to the capacitance plates on the other side of the detector. This involves a more elaborate, double-shielding arrangement, and a means of overcoming a capacitance coupling in the transformer.

\footnotetext{
1 Figures in brackets indicate the literature references at the end of this paper.
}

\section{Transformer Ratio-Arm Capacitance Bridge}

Figure 1 is a simplified schematic of the modified bridge, without the primary windings. There are two segments of the secondary windings for the ratio
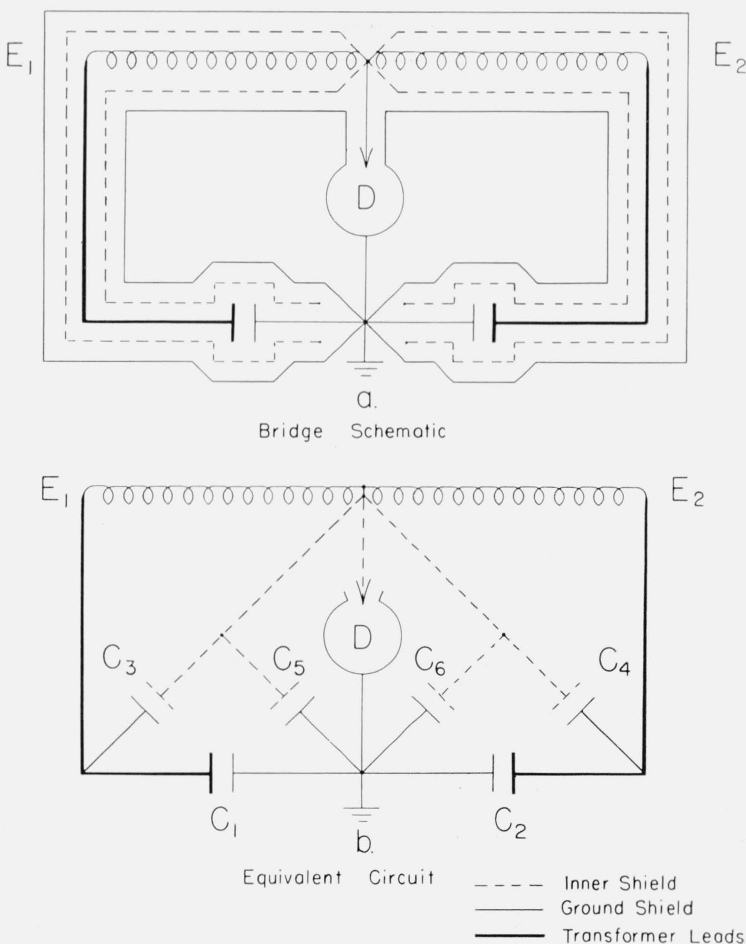

Figure 1. Bridge schematic. 
arms and the active elements of two three-lead capacitors, $\mathrm{C}_{1}$ and $\mathrm{C}_{2}$, for the other arms. A high impedance detector, $\mathrm{D}$, is connected from the center tap of the secondary windings to the grounded midpoint of the two capacitors. An inner shield, indicated in figure 1 by the dashed line, connects with the center-tap of the secondary windings and encloses the secondary windings and the capacitors. The capacitances, $\mathrm{C}_{3}$ and $\mathrm{C}_{4}$, between this shield and the transformer leads, are shunts which load both the leads and the ratio-arms, but are not detected as part of the measured capacitance. These capacitances are kept small external to the transformer and of approximately equal value so that the voltage drop in the lead of either secondary ratio arm, because of this additional loading, is less than 1 part in $10^{7}$, and is partially compensated as well by a similar shunting capacitance on the lead of the other ratio arm. An outer shield connected to the orounded midpoint between the two capacitors is necessary to reduce noise pickup by the system. As shown in figure 1 , the capacitances, $\mathrm{C}_{5}$ and $\mathrm{C}_{6}$, between this ground shield and the inner shield, shunt the detector and directly affect its sensitivity. 'To minimize this detector shunting capacitance, the ground shield is kept at as large a distance as possible from the inner shield.

The special construction of the transformer gives two voltages at $E_{1}$ and $E_{2}$, opposite in phase and of a fixed voltage ratio within a part per million of the turns ratio. This is accomplished by the use of tightly coupled secondary turns wound on a large toroidal core of high permeability tape. The two secondary windings are identical copper helices, constructed so as to have large cross section and low resistance and interleaved to sample very nearly the same flux along the core. The low resistance and leakage inductance of each secondary winding assure adequate voltage constancy at $E_{1}$ and $E_{2}$ when $\mathrm{C}_{3}$ does not vary from $\mathrm{C}_{4}$ by more than $1000 \mathrm{pF}$.

The properties of 3 -lead capacitors $[5,6]$ made it possible to define precisely small changes of capacitance $\left(1 \times 10^{-5} \mathrm{pF}\right)$ in this system. The bridge circuit, shown in figure 1 , eliminates the detection of parasitic capacitance, so that additional capacitors can be switched into the circuit in parallel with a reproducible and calculable effect.

Most components of the bridge were placed in a large metal enclosure at ground potential. Triaxial cable or a double shielding arrangement was necessary for each bridge element, such as a measuring probe, outside of the shielded enclosure. The use of a solid inner shield was found to be necessary to eliminate errors caused by the detection of parasitic capacitance.

\section{Transformer Design and Construction}

The specific design of the transformer with secondary windings in the ratio of 1 to 1 , is based on a suggestion by R. D. Cutkosky. ${ }^{2}$ An additional tap gives a second ratio of 4 to 3 .

\footnotetext{
2 Electricity Division, National Bureau of Standards.
}

The transformer is operated at a frequency of 1000 $\mathrm{Hz}$ with $40 \mathrm{~V}$ on the primary, which gives approximately $12 \mathrm{~V}$ across the secondary windings in series.

The primary winding consists of 132 turns of AWG No. 15 copper wire, closely and evenly spaced around the core of the transformer. To be sure of uniformity of spacing, a second Nylon form milled with grooves to receive the primary winding, was made to fit around the Nylon case of the core. One end of the primary was soldered to a smaller, more flexible wire which was placed around the outer circumference of the core in the direction opposite to the advance of the windings in order to compensate for the single turn effect caused by the advance of the main windings around the core. The core of the transformer is a toroid of $0.002 \mathrm{in}$. thick Supermalloy ${ }^{3}$ tape with an outer Nylon case 4.5 in. o.d., 3 in. i.d., and 1.5 in. high.

The bridge arrangement necessitates the use of two electrostatic shields between the primary and secondary windings. (See fig. 2.) These shields are part of the inner and ground shields referred to in the discussion of the bridge. Part of the ground shield encloses the primary windings and part of the inner shield encloses the secondary windings. That part of the ground shield enclosing the primary windings is in the shape of a toroidal cup of $1 / 16$ in. thick copper. A top cover to complete the shield is screwed to the outer circumference. A small gap in the shield, centered on the inside, prevents a shorted turn around the core of the transformer. A threaded ring, TR, attached to the shield on the top side of the gap can be moved up or down by rotation to change the "effective" gap position. Melamine sheet of 0.015 in. thickness is used as insulation between the primary windings and this shield. The need for the adjustable gap position will be explained later.

Each of the two secondary windings was constructed of 20 pieces of 0.025 in. thick copper sheet, machined in circular form, 5.5 in. o.d. and 3.5 in. i.d. The pieces were cut on a radius, properly oriented on a jig, and butt silver soldered to one another to form a helix. Two approximately identical secondary windings of 20 turns apiece were then, in effect, screwed together and insulated from one another by the insertion of 0.0075 in. thick Teflon sheets. Small flexible wires were used to make the connections from the windings to the BNC terminals mounted on the (enclosing) inner shield.

That part of the inner shield enclosing the secondary windings was assembled from 14 different copper pieces. They were accurately machined so they would fit tightly together with a steplike gap at the center of one side and located to be at about the same level as the gap of the primary shield. Four machined Nylon spacers were used to insulate the inner shield from the ground shield and to hold it in a stable position. The form and placement of the inner shield allowed comparatively little surface area close to the ground shield and thus the detector shunting capacitance was minimized.

\footnotetext{
${ }^{3}$ Arnold Engineering Company, Marengo, Ill.
} 


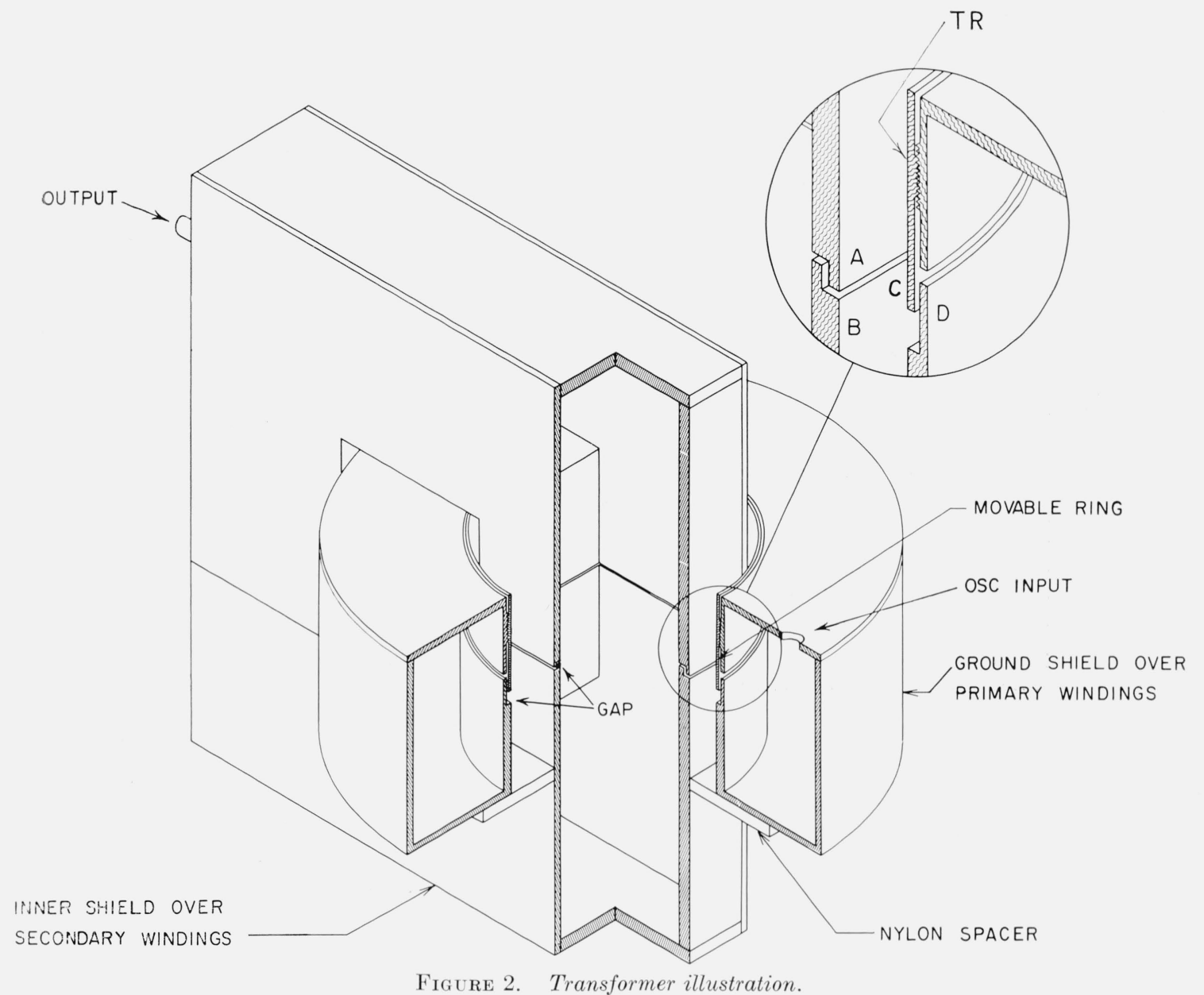

\section{Bridge Unbalance Due to Shield Arrangement}

Since both shields make one turn through the core of the transformer, the detector, connected across the shields, is influenced by the induced voltages on the shields. In the design being discussed the voltage across the gap of each shield is approximately 0.8 percent of the primary voltage. A current path is provided through the detector by capacitances between the secondary shield and the primary shield or ground. This can be explained further by reference to figures 2 and 3, which represent a sectional view showing the core, the primary shield and the secondary shield of the transformer and the equivalent electrical circuit. The windings are not shown and for the following discussion are not considered except to assume that the primary is providing flux to the core. When a positive voltage is induced at $\mathrm{A}$ (secondary) and $\mathrm{C}$ (primary), a negative voltage exists at B (secondary) and D (primary). The gap in each shield limits the current circulating within the shield. Because of equal voltage between A and $\mathrm{C}$ and between $\mathrm{B}$ and $\mathrm{D}$, only those capacitances between $\mathrm{A}$ and $\mathrm{D}$ and between $\mathrm{B}$ and $\mathrm{C}$ are important. A difference in the value of these capaci- tances creates a net current through the detector. A provision to reduce the current flow to zero by adjustment of these capacitances was made by the construction of a threaded ring attached to the inner wall of the primary shield.

The location of both gaps at the center of the core provides symmetry and reduces the effect of capacitance to the surroundings.

\section{Capacitance and Conductance Balance}

In almost all a-c bridges, means must be provided to balance out the resistive component of the system as well as the capacitive component in order to obtain a true null. This is accomplished by switching into one arm of the bridge or the other a conductive balance control (see fig. 4) to balance the excess resistive component in the adjacent arm of the bridge. The construction of a network to obtain a small voltage in phase with the voltage $E$, is described by Thompson [6] and others. An adaptation of this circuit has been made with only the components needed for operation at $1 \mathrm{kHz}$. A decadeswitched, tapped inductor connected across one secondary winding or the other acts as a voltage divider. The line from the tapped inductor is con- 


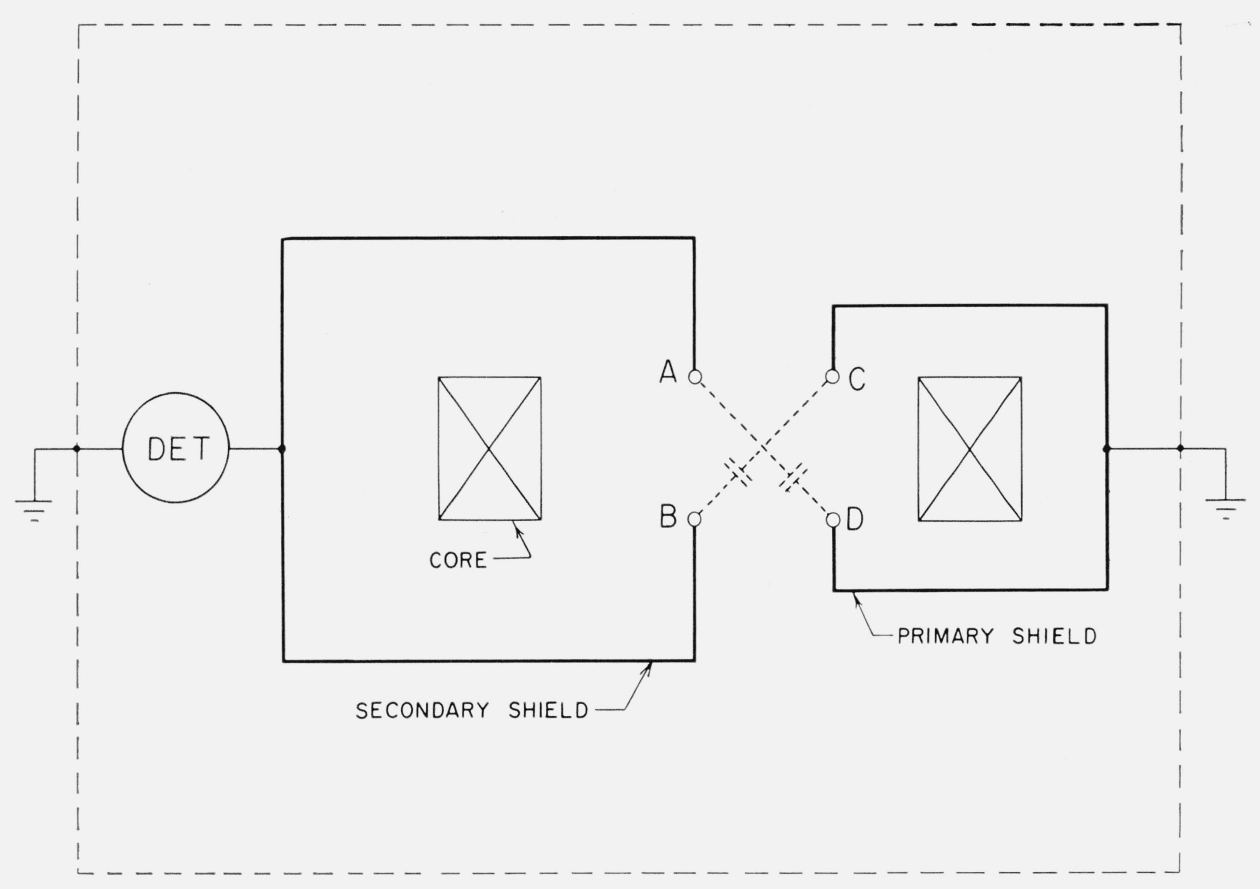

Figure 3. Shield arrangement.

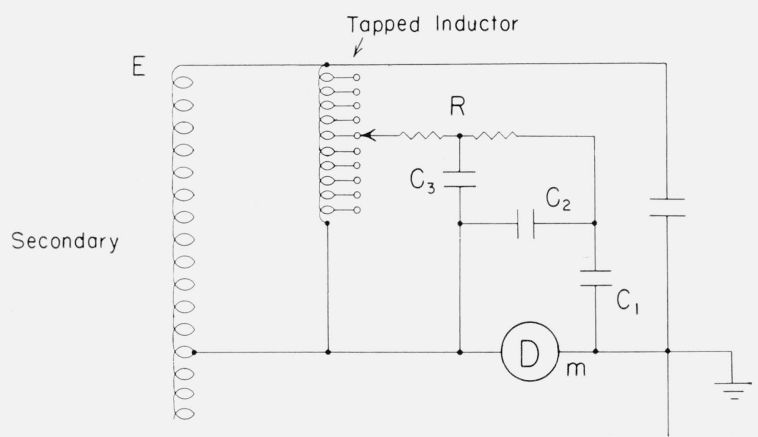

Figure 4. Conductive balance control.

nected to a "T" network consisting of a resistance with a midpoint connection and several capacitors. The values of $\mathrm{R}$ and $\mathrm{C}_{1}, \mathrm{C}_{2}$, and $\mathrm{C}_{3}$ are selected to produce a small voltage of correct magnitude and phase at the input to the detector. In effect the reactance of the combination of $\mathrm{C}_{1} \mathrm{C}_{2}$ in parallel is small compared to $\mathrm{R}$ and the voltage at $m$ due to this network is nearly in phase with voltage, $E$. It is made almost, if not exactly equal in phase by the small capacitance at $\mathrm{C}_{3}$.

\section{Shielded Switch}

To switch capacitors into or out of the bridge either singly or in combination, a modification to provide the proper shielding was made of a standard Leeds and Northrup 12-position silver contact rotary switch.

Figure 5 shows the terminals around the circumference of the switch laid out in the plane of the paper for ease of illustration. The heavy dark line, around the switch represents the inner shield, enclosing the entire switch.

The two portions of the shield represented by the horizontal lines were machined from brass to replace the original sections of the switch. Connections from the various switch terminals were made to $\mathrm{BNC}$ receptacles mounted through the shield. The common terminals $R_{1}$ and $R_{2}$ were connected to one of the ratio arms, and common terminal $\mathrm{L}$ was connected to the other ratio arm. Only 6 positions were used and certain brushes which connect some of the single terminals to a common terminal were relocated $180 \mathrm{deg}$ from the normal position.

Each position of the switch allows the connection of one or two of five different capacitance elements into one arm of the bridge at the same time connecting one or two of the remaining capacitance elements into the other arm. Any elements not being: used are connected to the shield so that their presence will not influence the balance point by parasitic capacitance. The lower portion of the switch is an arrangement to short out the input to the detector while going from one switch position to another.

\section{Operation of Bridge}

The ratio of the secondary voltages of the transformer used in the configuration described by Thompson is equal to the turns ratio within 1 or 2 parts in $10^{7}$. The modified bridge configuration loses this desirable feature because of the voltage produced on the secondary shield. 


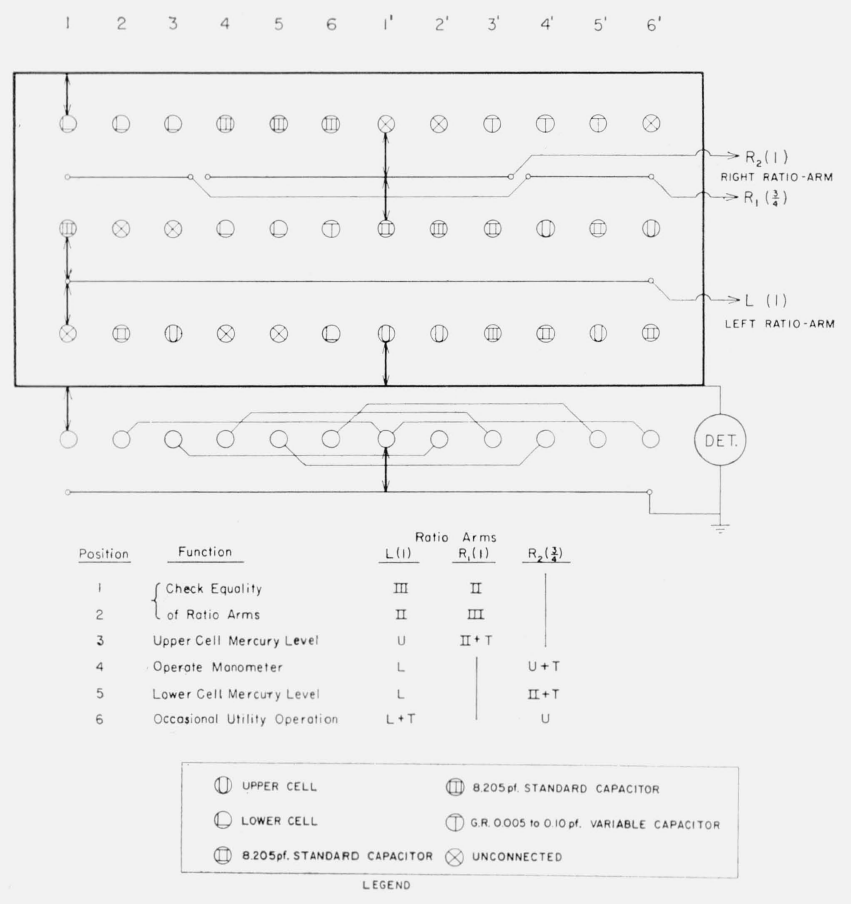

Figure 5. Coaxial switch.

Equality of the secondary voltages is tested by switching the ground point to the center tap of the secondary windings, so that the detectible effect of the shield capacitances is eliminated. For equal ratio arm voltages two nominally equal and highly stable standard capacitors ${ }^{4}$ are then placed in the arms of the bridge. The capacitance value of one is decreased to be equal within $1 \times 10^{-5} \mathrm{pF}$ of the value of the other by removal of a small area of the plate of the capacitor. A check of both the equality of the ratio arms and the equality of the capacitors is made by interchanging the capacitors in the arms.

The two equal standard capacitors are then used in the two equal arms of the modified arrangement and the movable inner ring of the primary shield is adjusted until null is again observed.

The complete detection system of the bridge and other components is illustrated in the block diagram of figure 6 . If desired, the output signal of the phase detector can be used for automatic control purposes.

Performance of the particular system described, over a period of several months, indicates that the standard capacitors have remained equal in value (within $1 \times 10^{-5} \mathrm{pF}$ ), and the shield cross capacitances have not changed sufficiently to introduce a significant error.

\footnotetext{
4 The values of the standard capacitors were made to be $8.205 \mathrm{pF}$. These capacitors were made from disks of high quality fused silica with a gold film eva porated onto polished surfaces to produce the plates and guard.
}

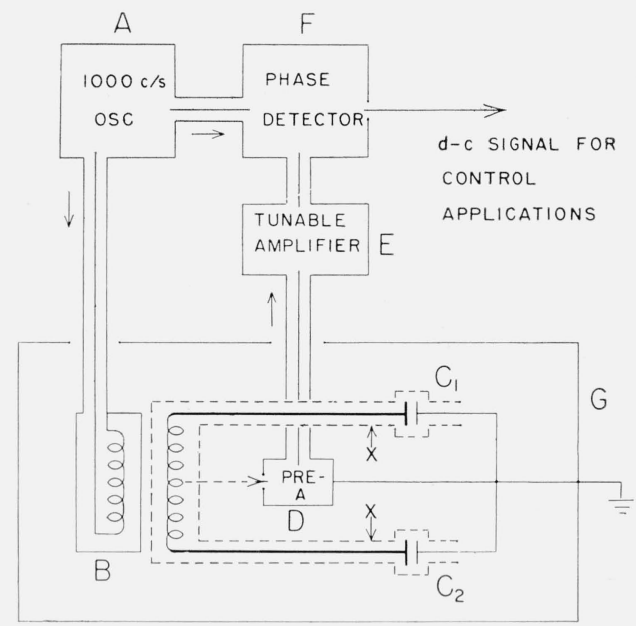

Figure 6. Block diagram of system.

\section{Determination of Probe or Cell Capacitance Constant}

In applications involving use of this bridge to determine the change of position of a grounded object, it is necessary to know the relation for the capacitance between the capacitance probe and the object. One may measure the mechanical dimensions of the capacitance detecting plate, shield ring, and reference object, and calculate capacitance versus the distance of separation of the reference object from the probe. Accuracy of the result is limited to the exactness of the capacitance equation available for the configuration used and the accuracy by which the mechanical dimensions of the probe can be measured. Exact equations are not available for the capacitance of some probe configurations. In these cases, a graphical relaxation method [8] of determining the potential field and thereby the capacitance can be used.

\section{Applications}

One application of the modified bridge is its use in a precision mercury manometer. In normal operation, the upper cell (see fig. 7) of the manometer is in one arm of the bridge and the lower cell is in the other. A 3-lead adjustable trimmer capacitor is in parallel with the lower cell. At "zero level" with both cells evacuated, the plate-to-mercury capacitance is not exactly the same in the two cells because of small differences in level of the plates, and differences in the mechanical configuration of the plate-guard assembly. This is compensated for by the trimmer. Subsequently the upper cell is wrung to the top of a column of gage blocks. When the pressure is such as to reestablish bridge null, the difference between separation of the upper cell mercury meniscus from its plate and the separation of the lower cell mercury meniscus from its plate 
is the same as zero level. Therefore the height of the column of mercury supported by the pressure in the lower cell is equal to the additional length of gage blocks placed between the upper cell and the base. Since the separation of the mercury from the

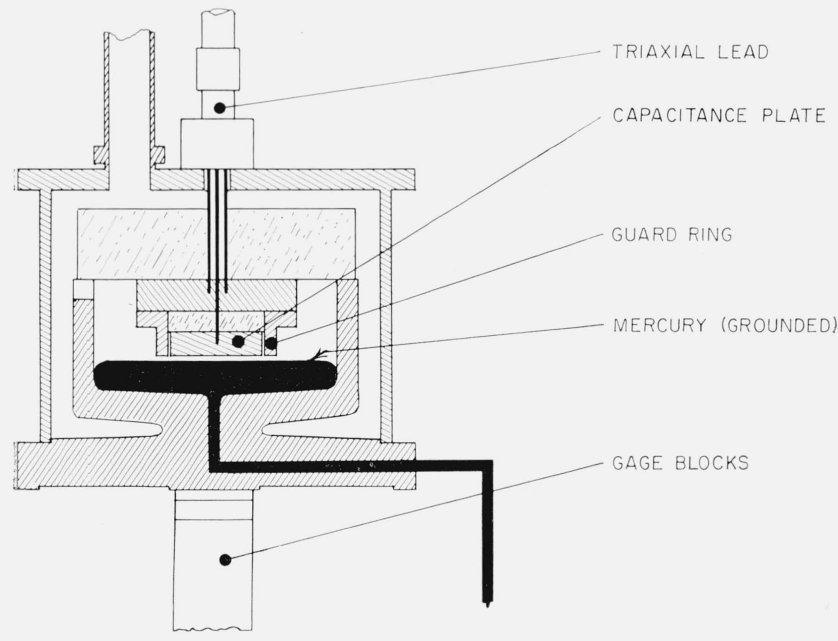

FiguRE 7. Mercury meniscus an l capacitance unit of manometer. plate is probably not exactly the same in the two cells, an error may result if the amount of the separation changes. The mercury level is adjusted so that the capacitance in the upper cell corresponds to about $800 \mu$ separation and the lower cell separation at bridge null is not more than $1 \mu$ different. So long as the separation in the upper cell does not vary more than $2 \mu$, the bridge null corresponds to the same pressure within $5 \mathrm{~m} \mu$. The total error in the pressure contributed by the detection system is in the millimicron range.

A second application for the modified bridge is illustrated by the determination of the inside diameter of a long metal tube, nominally $0.8 \mathrm{~mm}$. It is desired that the uncertainty in the inside diameter not exceed $6 \times 10^{-5} \mathrm{~cm}$. As shown in figure 8 , a small probe, $0.75 \mathrm{~mm}$ in diameter and $1 \mathrm{~mm}$ long, properly insulated and centered by air flow, is moved along the length of the tube to sense the capacitance with relation to the position of the probe. Other probes of 2 and $3 \mathrm{~mm}$ length and of the same diameter are used, so that capacitance "end effects" can be eliminated. It is especially convenient that the measured tube can be grounded, because the tube itself then serves as a shield for the rest of the system.

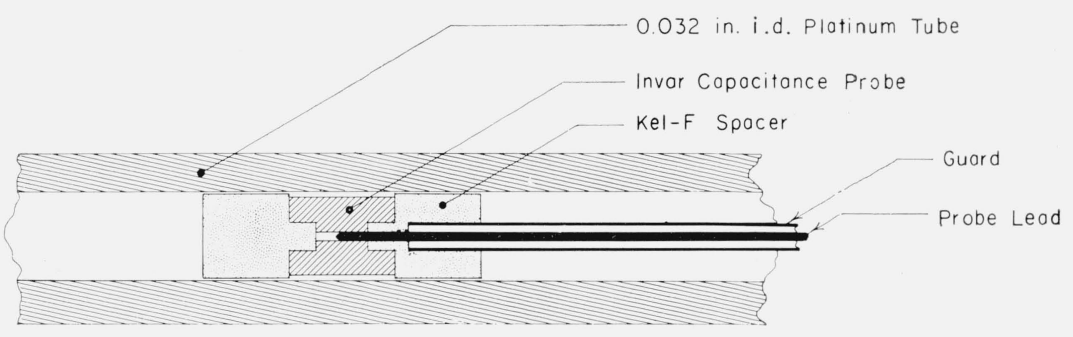

Figure 8. Small capacitance probe.

\section{References}

[1] A. D. Blumlein, British Patent No. 323037.

[2] H. A. M. Clark and P. B. Vanderlyn, Pro. Inst. Elec. Eng. 96, 365 (1949).

13] C. W. Oakley and J. G. Yates, Proc. Elect. Eng. 101, 91 (1954).

[4] C. H. Young, Bell Lab. Record 24, 433 (1946).

[5] F. K. Harris, Electrical Measurements, p. 682 (John Wiley and Sons, Inc., New York, N.Y., 1952).
[6] A. M. Thompson, Inst. Rad. Eng. Trans. on Instrumentation I- $\boldsymbol{\gamma}, 245$ (1958).

[7] M. C. McGregor, J. F. Hersh, R. D. Cutkosky, F. K. Harris, and F. R. Kotter, Inst. Rad. Eng. Trans. on Instrumentation I- $\mathbf{7}, 253$ (1958).

[8] D. N. de G. Allen, Relaxation Methods (McGraw-Hill Book Co., Inc., New York, 1954).

(Paper 69C1-180) 